\title{
Coherence Dynamics of Two Interacting Bosonic Modes in a Thermal Environment
}

\author{
Aurelian Isar ${ }^{1,2,3, \star}$ \\ ${ }^{1}$ Department of Theoretical Physics, National Institute of Physics and Nuclear Engineering, \\ 077125 Bucharest-Magurele, Romania \\ ${ }^{2}$ Faculty of Physics, University of Bucharest, 077125 Bucharest-Magurele, Romania \\ ${ }^{3}$ Academy of Romanian Scientists, 3 llfov str., 050044 Bucharest, Romania
}

\begin{abstract}
.
We describe the time evolution of the quantum coherence in an open system consisting of two coupled bosonic modes embedded in a thermal reservoir. We discuss the influence of the environment in terms of the covariance matrix for initial squeezed thermal states. The coherence is quantified using the relative entropy as a measure, and its dynamics is studied in the framework of the theory of open systems based on completely positive quantum dynamical semigroups. We show that the evolution of the quantum coherence strongly depends on the initial state of the system (squeezing parameter and thermal photon numbers), the parameters characterizing the thermal reservoir (temperature and dissipation coefficient) and the intensity of the coupling between the two modes.
\end{abstract}

\section{Introduction}

Quantum coherence arises from the superposition principle, which represents the essential point of departure of quantum physics from the classical views of the reality. Presently coherence is identified as a quantum resource for applications in science and quantum technologies [1,2]. Resource theories provide a powerful framework for the understanding of quantum physical phenomena, with fruitful applications in quantum information processing tasks [3, 4], quantum thermal engine [5, 6], quantum computing [7], cryptography [8], quantum metrology [9, 10] as well as in biological systems [11, 12].

Recently, Baumgratz et al. [13] proposed a framework for quantifying coherence, in which coherence is considered a quantum resource, in a similar axiomatic way like the approach to the quantification of entanglement and other quantum correlations or types of nonclassicality [14-20].

Most of the measures of the quantum coherence assume a finite-dimensional Hilbert space, however, some recent papers introduced a resource-theoretic framework for coherence in continuous variable systems, and, in particular, coherence of Gaussian states has been investigated [21-23].

In order to obtain a realistic description of the quantum processes it is necessary to take decoherence and dissipation into consideration. Recently we studied, in the framework of the theory of open systems, the dynamics of quantum correlations of two, both uncoupled and coupled, bosonic modes embedded in a common thermal bath, for initial Gaussian states of the system [24-30].

^e-mail: isar@theory.nipne.ro 
In this work we use the axiomatic formalism of the theory of open quantum systems based on completely positive dynamical semigroups [31] to address the quantification of the coherence, following Ref. [22], for an open system consisting of two coupled bosonic modes embedded in a thermal environment. We study the time evolution of the coherence of the system and show that it has a strong dependence on the initial state and the parameters characterizing the open system and the environment. Previously we described the evolution of quantum coherence of two uncoupled bosonic modes interacting with a thermal bath [32]. Likewise, in Refs. [33, 34] the authors considered a system of two non-interacting bosonic modes, but they addressed a more general scenario, including a nonclassical contribution from the environment.

The paper is organised as follows. In Sec. 2 we write the Markovian master equation for the density operator of the open system interacting with a general environment and solve the evolution equation for the covariance matrix of the state of the bimodal bosonic system [35]. In Sec. 3 we provide a coherence measure for Gaussian states based on the relative entropy. In Sec. 4 we describe the time evolution of the coherence of the open system of two coupled bosonic modes as a function of the parameters of the considered system and environment. The conclusions are given in Sec. 5 .

\section{Master equation for two bosonic modes interacting with the environment}

In order to study the dynamics of the subsystem consisting of two coupled bosonic modes (harmonic oscillators) weakly interacting with a thermal reservoir, we use the axiomatic formalism based on completely positive quantum dynamical semigroups. In this framework the Markovian irreversible time evolution of an open system is described by the following Gorini-Kossakowski-SudarshanLindblad master equation for the density operator $\rho(t)$ [31, 35-37]:

$$
\frac{d \rho(t)}{d t}=-\frac{i}{\hbar}[H, \rho(t)]+\frac{1}{2 \hbar} \sum_{n}\left(2 B_{n} \rho(t) B_{n}^{\dagger}-\left\{\rho(t), B_{n}^{\dagger} B_{n}\right\}_{+}\right),
$$

where $H$ denotes the Hamiltonian of the open system and the operators $B_{n}, B_{n}^{\dagger}$ are defined on the Hilbert space of $H$, and describe the interaction of the subsystem with a general environment.

The Hamiltonian of two coupled in coordinates non-resonant harmonic oscillators of identical mass $m$ and frequencies $\omega_{1}$ and $\omega_{2}$ is given by

$$
H=\frac{1}{2 m}\left(p_{x}^{2}+p_{y}^{2}\right)+\frac{m}{2}\left(\omega_{1}^{2} x^{2}+\omega_{2}^{2} y^{2}\right)+q x y,
$$

where $x, y$ are the coordinates and $p_{x}, p_{y}$ are the momenta of the two modes, $q$ is the coupling parameter, and we take the operators $B_{j}$ as polynomials of first degree in the canonical variables of coordinates and momenta.

The equations of motion for the quantum correlations of the canonical observables are the following ( $\mathrm{T}$ denotes the transposed matrix) [35]:

$$
\frac{d \sigma(t)}{d t}=Z \sigma(t)+\sigma(t) Z^{\mathrm{T}}+2 D, \quad Z=\left(\begin{array}{cccc}
-\lambda & 1 / m & 0 & 0 \\
-m \omega_{1}^{2} & -\lambda & -q & 0 \\
0 & 0 & -\lambda & 1 / m \\
-q & 0 & -m \omega_{2}^{2} & -\lambda
\end{array}\right)
$$

with real diffusion matrix $D$ and dissipation coefficient $\lambda$. We introduced the $4 \times 4$ bimodal covariance matrix $\sigma$, with the elements defined as $\sigma_{i j}=\operatorname{Tr}\left[\left(A_{i} A_{j}+A_{j} A_{i}\right) \rho\right], i, j=1, \ldots, 4$, with $\mathbf{A}=\left\{x, p_{x}, y, p_{y}\right\}$, which fully characterize any Gaussian state of a bimodal system (up to local displacements). 
The time-dependent solution of Eq. (3) is given by [35]

$$
\sigma(t)=S(t)[\sigma(0)-\sigma(\infty)] S^{\mathrm{T}}(t)+\sigma(\infty),
$$

where $S(t) \equiv \exp (Z t)$. The values at infinity are obtained from the equation $Z \sigma(\infty)+\sigma(\infty) Z^{\mathrm{T}}=-2 D$. The covariance matrix, which is a real, symmetric and positive matrix entirely specifying a two-mode Gaussian state, has the following block structure, where $2 \times 2$ Hermitian matrices $A$ and $B$ are the covariance matrices for the single modes, and $C$ contains the cross-correlations between the modes:

$$
\sigma(t)=\left(\begin{array}{cc}
A & C \\
C^{\mathrm{T}} & B
\end{array}\right)
$$

\section{Coherence of Gaussian states}

A state is called incoherent if it is diagonal in a fixed orthonormal basis. We denote the set of all incoherent states by $\mathcal{I}$. In Ref. [13] there have been proposed the following postulates that any proper measure of the coherence $C(\rho)$ for finite-dimensional states $\rho$ must satisfy.

(C1) $C(\rho) \geq 0$ and $C(\delta)=0$ if and only if $\rho \in \mathcal{I}$.

(C2a) Monotonicity under all incoherent completely positive and trace-preserving (ICPTP) maps: $C(\rho) \geq C\left(\Phi_{\text {ICPTP }}(\rho)\right)$, where $\Phi_{\text {ICPTP }}(\rho)=\sum_{n} K_{n} \rho K_{n}^{\dagger}, \sum_{n} K_{n}^{\dagger} K_{n}=I, K_{n} \mathcal{I} K_{n}^{\dagger} \subset \mathcal{I}$ for all $n$, with $\dagger$ the adjoint and $I$ identity operator.

(C2b) Monotonicity for average coherence under the subselection based on measurement outcomes: $C(\rho) \geq \sum_{n} p_{n} C\left(\rho_{n}\right)$, where $\rho_{n}=K_{n} \rho K_{n}^{\dagger} / p_{n}$ and $p_{n}=\operatorname{Tr}\left(K_{n} \rho K_{n}^{\dagger}\right), \sum_{n} K_{n}^{\dagger} K_{n}=I, K_{n} \mathcal{I} K_{n}^{\dagger} \subset \mathcal{I}$ for all $n$.

(C3) Nonincreasing under the mixing of quantum states: $\sum_{n} p_{n} C\left(\rho_{n}\right) \geq C\left(\sum_{n} p_{n} \rho_{n}\right)$.

We note that $(\mathrm{C} 2 \mathrm{~b})$ and $(\mathrm{C} 3)$ together imply $\mathrm{C}(2 \mathrm{a})$.

In Ref. [22] $\mathrm{Xu}$ gives the following definition of an incoherent Gaussian channel (IGC): it is a Gaussian channel that maps thermal states into thermal states. Instead of $(\mathrm{C} 2 \mathrm{a})$ and $(\mathrm{C} 2 \mathrm{~b})$ it is required that any coherence measure $C$ for Gaussian states satisfies

(C2) $C(\rho) \geq C\left(\Phi_{\mathrm{IGC}}(\rho)\right)$ for any Gaussian state $\rho$ and any incoherent Gaussian channel IGC.

The coherence measure for Gaussian states

$$
C(\rho)=\inf _{\delta}\{S(\rho \| \delta): \delta \text { is an incoherent Gaussian state }\},
$$

where inf runs over all incoherent Gaussian states, is given in terms of the relative entropy for two density matrices $\rho$ and $\delta$ :

$$
S(\rho \| \delta)=\operatorname{Tr}\left(\rho \log _{2} \rho\right)-\operatorname{Tr}\left(\rho \log _{2} \delta\right) .
$$

Here the first term represents the von Neumann entropy of $\rho, S(\rho)=-\operatorname{Tr}\left(\rho \log _{2} \rho\right)$. In the case of a bipartite system we have

$$
S(\rho)=-\sum_{i=1}^{2}\left(\frac{k_{i}-1}{2} \log _{2} \frac{k_{i}-1}{2}-\frac{k_{i}+1}{2} \log _{2} \frac{k_{i}+1}{2}\right),
$$

where $k_{1}, k_{2}$ are the symplectic eigenvalues of the covariance matrix $\sigma(t)$ defined as [38]

$$
2 k_{1,2}^{2}=\Delta \mp \sqrt{\Delta^{2}-4 \operatorname{det} \sigma(t)}
$$

and $\Delta$ is the symplectic invariant (seralian) $\Delta=\operatorname{det} A+\operatorname{det} B+2 \operatorname{det} C$. 
Using the definition (6) of the coherence, one obtains the following expression of the relative entropy of the coherence for Gaussian states [22]:

$$
C(\rho)=\inf _{\delta}\left[-S(\rho)-\operatorname{Tr}\left(\rho \log _{2} \delta\right)\right]=-S(\rho)+\sup _{\delta} \operatorname{Tr}\left(\rho \log _{2} \delta\right)=S\left(\rho_{\text {diag }}\right)-S(\rho),
$$

where $\rho_{\text {diag }}$ is the density matrix of the closest Gaussian thermal state to the given state $\rho$ and has a covariance matrix described by the average number of thermal photons

$$
n_{1}^{\text {th }}=\frac{1}{4}\left(A_{11}+A_{22}-2\right), \quad n_{2}^{\text {th }}=\frac{1}{4}\left(B_{11}+B_{22}-2\right) .
$$

Thus, the final expression for the relative entropy of coherence is

$$
C(\rho)=\sum_{i=1}^{2}\left[g\left(2 n_{i}^{\mathrm{th}}+1\right)-g\left(k_{i}\right)\right],
$$

with

$$
g(x)=\frac{x+1}{2} \log _{2} \frac{x+1}{2}-\frac{x-1}{2} \log _{2} \frac{x-1}{2}
$$

\section{Time evolution of the quantum coherence}

We take an initial two-mode squeezed thermal state, with the covariance matrix given by [39]

$$
\sigma_{s t}(0)=\left(\begin{array}{cccc}
a & 0 & c & 0 \\
0 & a & 0 & -c \\
c & 0 & b & 0 \\
0 & -c & 0 & b
\end{array}\right),
$$

where

$$
\begin{aligned}
& a=2 n_{1} \cosh ^{2} r+2 n_{2} \sinh ^{2} r+\cosh 2 r, \\
& b=2 n_{1} \sinh ^{2} r+2 n_{2} \cosh ^{2} r+\cosh 2 r, \\
& c=\left(n_{1}+n_{2}+1\right) \sinh 2 r .
\end{aligned}
$$

Here $n_{1}, n_{2}$ denote the mean thermal photon numbers associated with the two modes and $r$ is the squeezing parameter. For $n_{1}=0$ and $n_{2}=0$, (14) becomes the covariance matrix of the two-mode squeezed vacuum state.

We suppose that the only non-zero quantum diffusion coefficients have the following form (we put $m=\hbar=1)[31]$ :

$$
\omega_{1}^{2} D_{x x}=D_{p_{x} p_{x}}=\omega_{1} \lambda \operatorname{coth} \frac{\omega_{1}}{2 T}, \quad \omega_{2}^{2} D_{y y}=D_{p_{y} p_{y}}=\omega_{2} \lambda \operatorname{coth} \frac{\omega_{2}}{2 T},
$$

where $T$ is the temperature of the thermal reservoir (we have set the Boltzmann constant $k=1$ ).

We have analyzed the time evolution of the coherence of the two coupled bosons as a function of the squeezing parameter $r$, dissipation constant $\lambda$, coupling constant $q$ and temperature of the reservoir $T$ for fixed values of the average number of photons $n_{1}, n_{2}$ in the resonant case.

In Fig. 1 we plot the time variation of the coherence as a function of the squeezing parameter $r$. The relative entropy of coherence increases with the increase of the squeezing parameter, but has a slower increase at a higher temperature. The coherence decays asymptotically in time to small, but non-vanishing values, and presents a non-monotonic evolution. 

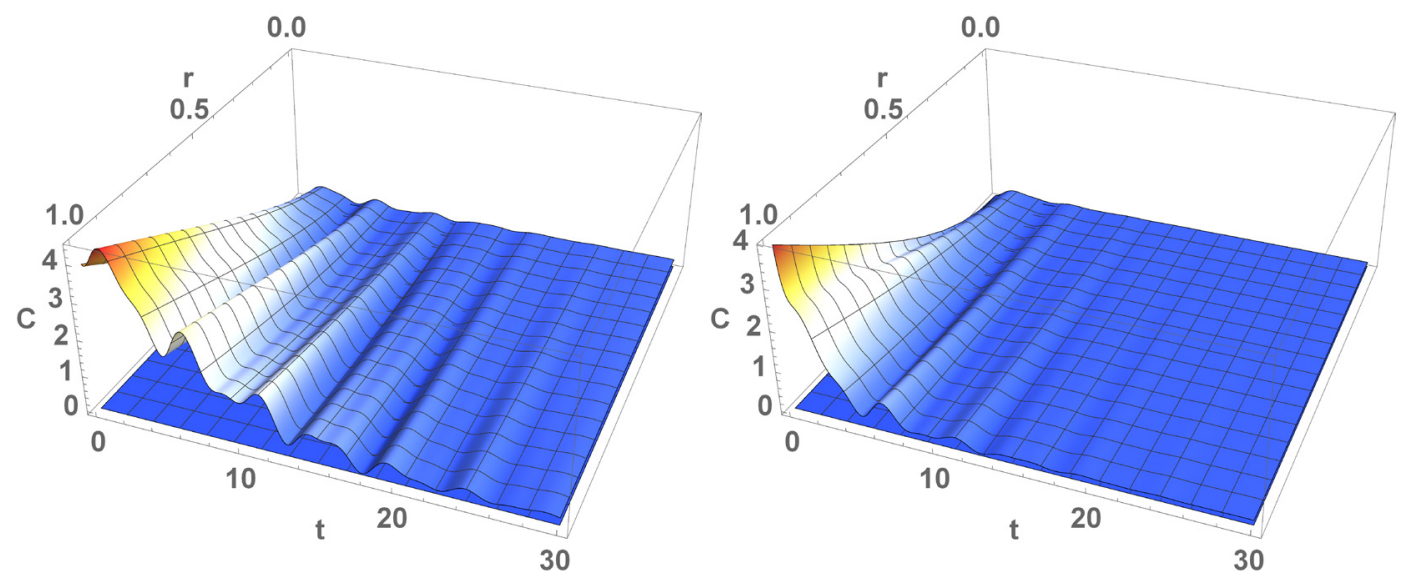

Figure 1. (Color online) Relative entropy of coherence $C$ for two bosonic modes with $n_{1}=1, n_{2}=2, \lambda=0.1$, $q=0.5$ as a function of time $t$ and squeezing parameter $r$ for the resonant case $\omega_{1}=\omega_{2}=1$ and for $T=0$ (left) and $T=5$ (right). Prevailing trends: Slow variation with $r$ at fixed $t$ (almost constant values at large $t$, slow to moderate decay at large $t$ ). Quasi-periodic variation with $t$ along constant $r$, with increasing amplitudes with $r$.
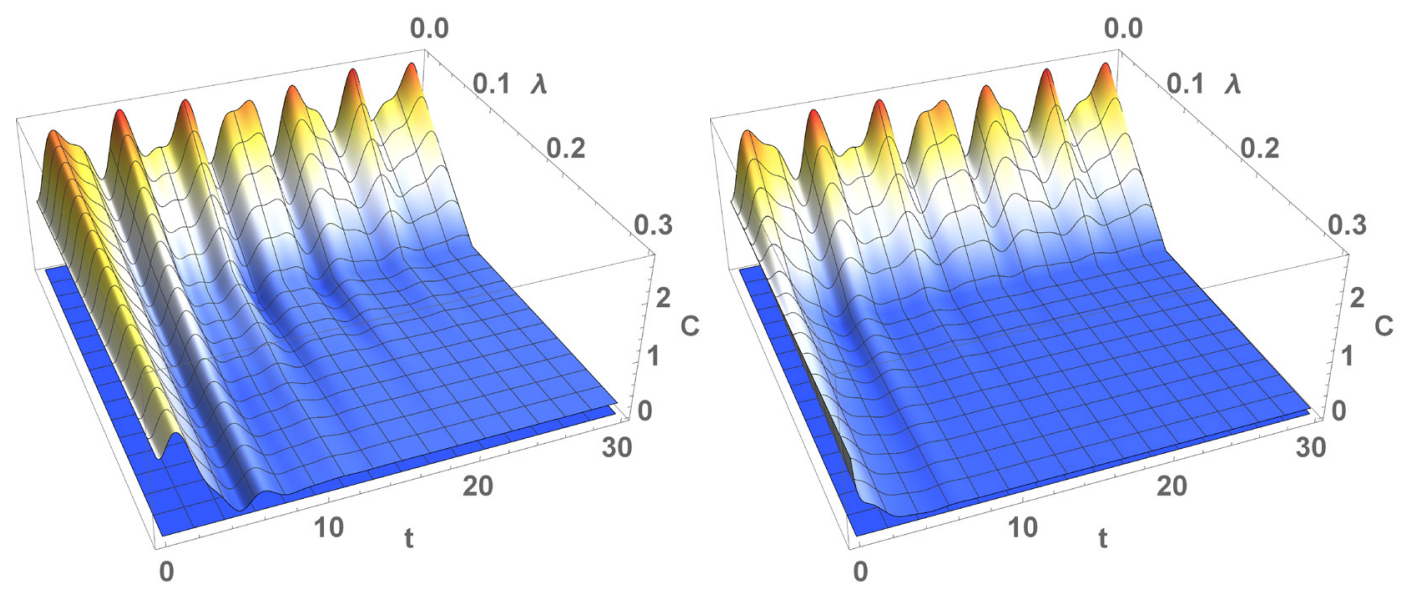

Figure 2. (Color online) Relative entropy of coherence $C$ for two bosonic modes with $n_{1}=1, n_{2}=2, r=0.5$, $q=0.5$ as a function of time $t$ and dissipation constant $\lambda$ for the resonant case $\omega_{1}=\omega_{2}=1$ and for $T=0$ (left) and $T=5$ (right). General trends: quasi-periodic variation with $t$ at fixed $\lambda$ (with more pronounced periodicity toward small $\lambda$ ), monotonically increasing variation under decreasing $\lambda$ at fixed $t$. 

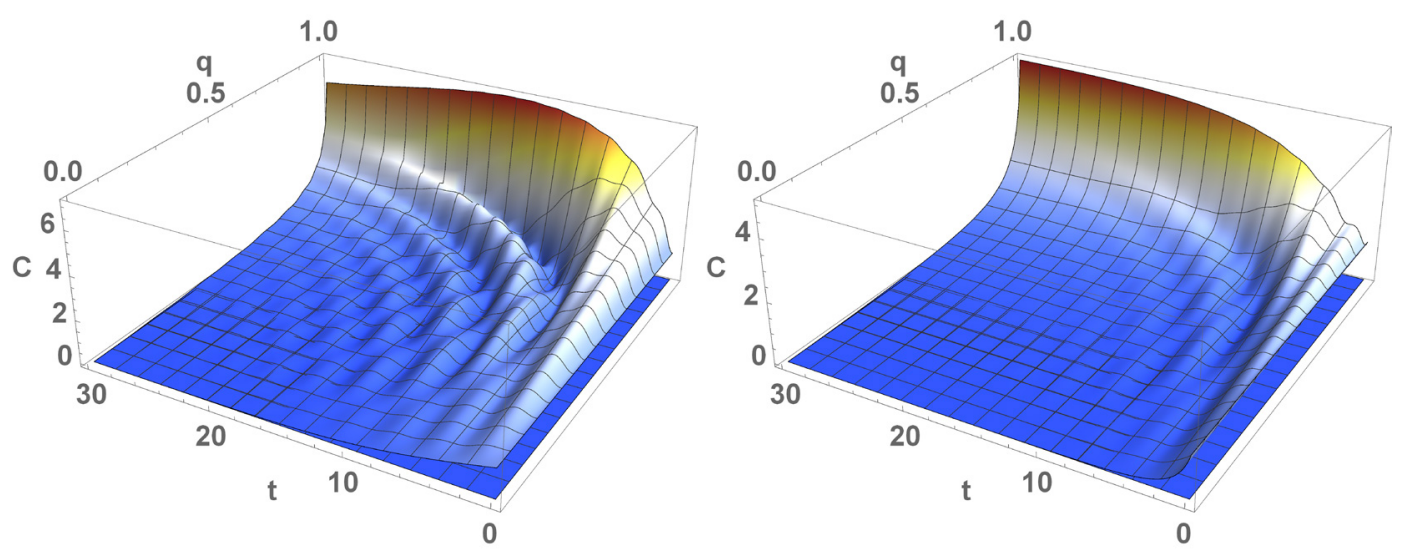

Figure 3. (Color online) Relative entropy of coherence $C$ for two bosonic modes with $n_{1}=1, n_{2}=2, r=0.5$, $\lambda=0.1$ as a function of time $t$ and coupling $q$ for the resonant case $\omega_{1}=\omega_{2}=1$ and for $T=0$ (left) and $T=5$ (right). Prevailing trend: radial wavy behaviour of increasing amplitude under increasing $q$ but decreasing $t$.

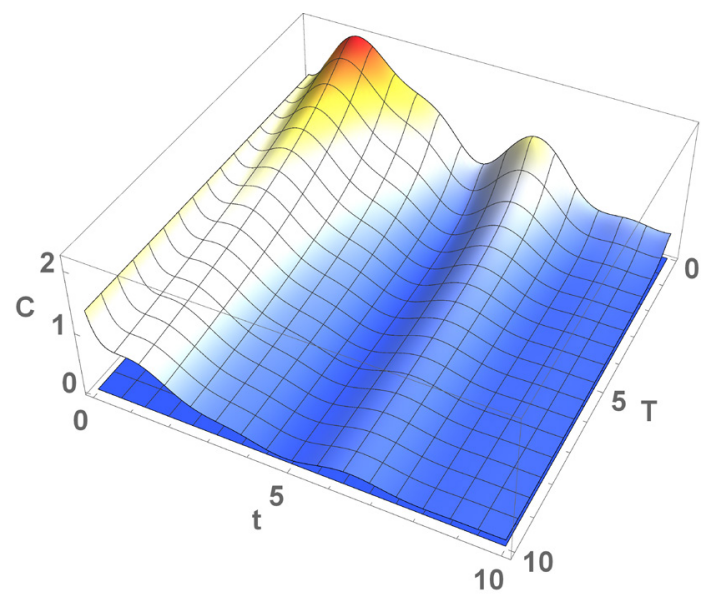

Figure 4. (Color online) Relative entropy of coherence $C$ for two bosonic modes with $n_{1}=1, n_{2}=2, r=0.5$, $\lambda=0.1, q=0.5$ as a function of time $t$ and temperature $T$ for the resonant case $\omega_{1}=\omega_{2}=1$. General trends: monotonic increase under decreasing $T$ at fixed $t$; wavy behaviour with $t$ at fixed $T$ (of increasing amplitude toward low $T$ ).

An almost periodic behaviour can be observed in the time evolution of the relative entropy of coherence as a function of the dissipation parameter $\lambda$. See Fig. 2, where we plot the coherence for fixed average thermal photon numbers, squeezing parameter and coupling constant, as a function of time and dissipation parameter $\lambda$. As expected, the coherence increases with the decrease of the dissipation constant and for small values of $\lambda$ fluctuates around positive values. 
One can observe the the relative entropy of coherence increases with the strength of the interaction between the bosonic modes (see Fig. 3) and decreases with the temperature of the environment (see Fig. 4). Therefore, in the resonant case it is possible to generate coherence in the system by increasing the coupling between the modes and decreasing the temperature. The direct interaction between the modes favours also the preservation in time of the coherence in the resonant case, as seen from Fig. 3.

\section{Summary and conclusions}

We have investigated the Markovian evolution of the quantum coherence of a system composed of two coupled bosonic modes embedded in a thermal environment using the axiomatic formalism based on completely positive quantum dynamical semigroups. The dynamics of the coherence is studied for an initial two-mode squeezed thermal state. We used the measure of coherence defined in terms of the relative entropy of the given state and of the closest Gaussian thermal state.

We have shown that the quantum coherence is increasing with the squeezing parameter of the initial state and the strength of the coupling between the modes, and it is decreasing by increasing the dissipation constant and temperature of the thermal bath. However, unlike in the previous study [32], where the system formed of two non-coupled bosonic modes in a thermal environment exhibited a quantum coherence asymptotically decreasing to zero, in the present model the quantum coherence tends to a non-zero asymptotic value, due to the interaction between the two bosonic modes, that sustains the coherence.

In the context of the debate relative to the physical interpretation of the quantum resources, the present results might be useful in controlling quantum coherence in open systems and also for applications in protocols of quantum information processing and communication.

\section{Acknowledgments}

The author acknowledges the financial support received from the Romanian Ministry of Research and Innovation, through the Project PN 190601 01/2019.

\section{References}

[1] A. Streltsov, G. Adesso, M. Plenio, Rev. Mod. Phys. 89, 041003 (2017)

[2] E. Chitambar, G. Gour, Rev. Mod. Phys. 91, 025001 (2019)

[3] R. Horodecki, P. Horodecki, M. Horodecki, K. Horodecki, Rev. Mod. Phys. 81, 865 (2009)

[4] H. Ollivier, W. H. Zurek, Phys. Rev. Lett. 88, 017901 (2001)

[5] M. O. Scully, M. S. Zubairy, G. S. Agarwal, H. Walther, Science 299, 862 (2003)

[6] M. O. Scully, K. R. Chapin, K. E. Dorfman, M. B. Kim, A. Svidzinsky, Proc. Nat. Acad. Sci. 108, 15097 (2011)

[7] T. D. Ladd, F. Jelezko, R. Laflamme, Y. Nakamura, C. Monroe, J. L. O’Brien, Nature 464, 45 (2010)

[8] V. Scarani, H. Bechmann-Pasquinucci, N. J. Cerf, M. Dusek, N. Lütkenhaus, M. Peev, Rev. Mod. Phys. 81, 1301 (2009)

[9] V. Giovannetti, S. Lloyd, L. Maccone, Nature Photonics 5, 222 (2011)

[10] G. Tóth, I. Apellaniz, J. Phys. A: Math. Theor. 47, 424006 (2014)

[11] S. Lloyd, J. Phys.: Conf. Series 302, 012037 (2011)

[12] G. S. Engel, T. R. Calhoun, E. L. Read, T. K. Ahn, T. Mancal, Y. C. Cheng, R. E. Blankenship, G. R. Fleming, Nature 446, 782 (2007) 
[13] T. Baumgratz, M. Cramer, M. B. Plenio, Phys. Rev. Lett. 113, 140401 (2014)

[14] V. Vedral, M. B. Plenio, M. A. Rippin, P. L. Knight, Phys. Rev. Lett. 78, 2275 (1997)

[15] V. Vedral, M. B. Plenio, Phys. Rev. A 57, 1619 (1998)

[16] A. Streltsov, U. Singh, H. S. Dhar, M. N. Bera, G. Adesso, Phys. Rev. Lett. 115, 020403 (2015)

[17] K. C. Tan, H. Jeong, Phys. Rev. Lett. 121, 220401 (2018)

[18] M.-L. Hu, X. Hu, J.-C. Wang, Y. Peng, Y.-R. Zhang, H. Fan, Phys Rep. 762, 1 (2018)

[19] X. Yuan, M.-A. Côté, A. Sordoni, R. Laroche, R. T. des Combes, M. Hausknecht, A. Trischler, arXiv:1706.04853 (2017)

[20] N. Killoran, F. E. S. Steinhoff, M. B. Plenio, Phys. Rev. Lett 116, 080402 (2016)

[21] Y.-R. Zhang, L.-H. Shao, Y. Li, H. Fan, Phys. Rev. A 93, 012334 (2016)

[22] J. Xu, Phys. Rev. A 93, 032111 (2016)

[23] D. Buono, G. Nocerino, G. Petrillo, G. Torre, G. Zonzo, F. Illuminati, arXiv:1609.00913 (2016)

[24] A. Isar, Phys. Scr. T 140, 014023 (2010)

[25] A. Isar, Phys. Scr. T 143, 014012 (2011)

[26] A. Isar, Phys. Scripta T 153, 014035 (2013)

[27] I. Ghiu, A. Isar, Rom. J. Phys. 61, 768 (2016)

[28] A. Isar, Open Sys. Information Dyn. 23, 1650007 (2016)

[29] A. Isar, T. Mihaescu, Eur. Phys. J. D 71, 144 (2017)

[30] T. Mihaescu, A. Isar, Eur. Phys. J. D 72, 104 (2018)

[31] A. Isar, A. Sandulescu, H. Scutaru, E. Stefanescu, W. Scheid, Int. J. Mod. Phys. E 3, 635 (1994)

[32] S. Suciu, A. Isar, Rom. J. Phys. 61, 1474 (2016)

[33] S. Olivares, M. G. A. Paris, R. Bonifacio, Phys. Rev. A 67, 032314 (2003)

[34] A. R. Rossi, S. Olivares, M. G. A. Paris, J. Mod. Optics 51, 1057 (2004)

[35] A. Sandulescu, H. Scutaru, W. Scheid, J. Phys. A: Math. Gen. 20, 2121 (1987)

[36] V. Gorini, A. Kossakowski, E. C. G. Sudarshan, J. Math. Phys. 17, 821 (1976)

[37] G. Lindblad, Commun. Math. Phys. 48, 119 (1976)

[38] G. Adesso, S. Ragy, A. R. Lee, Open Sys. Information Dyn. 21, 1440001 (2014)

[39] P. Marian, T. A. Marian, H. Scutaru, Phys. Rev. A 68, 062309 (2003) 